\title{
Comparison between Auditory and Visual Simple Reaction Times
}

\author{
Jose Shelton ${ }^{1}$, Gideon Praveen Kumar ${ }^{2}$ \\ ${ }^{1}$ Gippsland Physiotherapy Group, Melbourne, Australia; ${ }^{2}$ VIT University, Vellore, India. \\ Email: \{gideonpraveenkumar, josenoel2008\}@gmail.com
}

Received May 30 ${ }^{\text {th }}, 2010$; revised August $2^{\text {nd }}, 2010$; accepted August $7^{\text {th }}, 2010$.

\begin{abstract}
Objective: The purpose of this study was to find out whether the simple reaction time was faster for auditory or visual stimulus and the factors responsible for improving the performance of the athlete. Methodology: 14 subjects were assigned randomly into groups consisting of 2 members. Both the members from each group performed both the visual and auditory tests. The tests were taken from the DirectRT software program from a laptop. The DirectRT software consists of Testlabvisual and Testlabsounds to test the reaction times to visual and auditory stimuli. The 2 members from each group completed both the visual and auditory reaction times, the data was taken and the mean reaction time was calculated excluding the first and last values. Results: The results show that the mean visual reaction time is around 331 milliseconds as compared to the mean auditory reaction time of around 284 milliseconds. Conclusion: This shows that the auditory reaction time is faster than the visual reaction time. And also males have faster reaction times when compared to females for both auditory as well as visual stimuli.
\end{abstract}

Keywords: Reaction Time, Auditory Stimuli, Visual Stimuli, Neuromuscular-Physiological Response, Auditory Cortex, Visual Cortex, Muscle Contraction

\section{Introduction}

Reaction time (RT) is the elapsed time between the presentation of a sensory stimulus and the subsequent behavioral response. Simple reaction time is usually defined as the time required for an observer to detect the presence of a stimulus. It is a physical skill closely related to human performance. It represents the level of neuromuscular coordination in which the body through different physical, chemical and mechanical processes decodes visual or auditory stimuli which travel via afferent pathways and reach the brain as sensory stimuli.

Simple reaction time can be determined when an individual is asked to press a button as soon as a light or sound appears. Research done by Pain \& Hibbs, reference [1], shows that simple auditory reaction time has the fastest reaction time for any given stimulus. A study done by Thompson et al., reference [2] has documented that the mean reaction time to detect visual stimuli is approximately 180 to 200 milliseconds, whereas for sound it is around 140-160 milliseconds. On the other hand, there are also researches done by Yagi et al., reference [3], that show that reaction time to visual stimuli is faster than to auditory stimuli. Research by Verleger, reference [4] also confirms that visual reaction time is faster than auditory reaction time during or after exercise.

There are various factors that affect the reaction time to a stimulus. Factors like intensity and duration of the stimulus, age and gender of the participant, effect of practice can affect the reaction time of an individual to a particular stimulus. For example, there are relative differences between the reaction time to visual and auditory stimuli between genders. Male athletes tend to be faster than their female counterparts in responding to different stimuli. Researches done by Engel, reference [5], show the reaction time to sound to be faster in males when compared to females. Studies done by Dane et al., reference [6], show the difference in reaction time in eye-hand reaction time among male and female handball players.

The purpose of this study was to find out whether the simple reaction time was faster for auditory or visual stimulus and the factors responsible for improving the performance of the athlete.

\section{Methodology}

14 subjects were randomly divided into groups consisting 
of 2 members. Both the members from each group performed both the visual and auditory tests. The tests were taken from the DirectRT software program in the laptop. The tests for visual reaction time were taken from the 'testlabvisual' file in the DirectRT program. Before starting the test, the subjects were asked to give individual file numbers under the 'enter codes' menu, in order to access the data after the test. In the testlabvisual test, the subjects were asked to press the 'space bar' key, every time they saw a yellow box on the screen. Once the test was completed, the data was taken from the output file, the mean reaction time was calculated excluding the first and last values. After both the subjects from each group completed the visual test, they undertook the auditory reaction test. This was taken from the 'testlabsounds' file in the DirectRT program. In the testlabsounds test, the subjects were asked to press the 'spacebar' key, every time they heard a 'beep' sound. Once the test was completed, the data was taken from the output file, the mean reaction time was calculated excluding the first and last values. After both the members of a group completed the visual and auditory tests, the mean reaction time data for both the visual and auditory tests were entered in the laptop.

\section{Results}

The results show that the auditory reaction time is faster than the visual reaction time. And also males have faster reaction times when compared to females for both auditory as well as visual stimuli.

\section{Discussion}

As the result shows, in Figure 1, the mean visual reaction time is around 331 milliseconds as compared to the mean auditory reaction time of around 284 milliseconds. This confirms that the auditory reaction time is definitely faster compared to the visual reaction time. This finding is similar to the studies done by Pain \& Hibbs, reference [1]

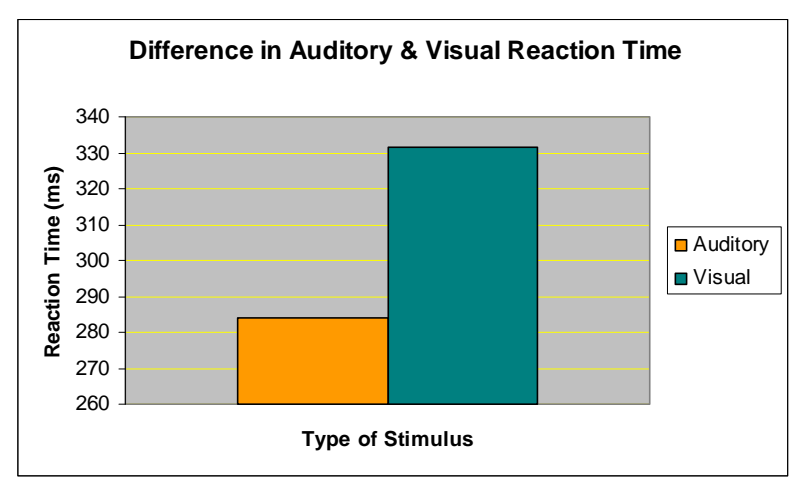

Figure 1. Graph showing faster simple reaction time for auditory stimulus compared to visual stimulus. and Thompson et al., reference [2], which also show that auditory reaction time is faster than visual reaction time.

Reaction time is dependent on several factors like arrival of the stimulus at the sensory organ, conversion of the stimulus by the sensory organ to a neural signal, neural transmissions and processing, muscular activation, soft tissue compliance, and the selection of an external measurement parameter (Pain \& Hibbs, reference [1]). Re- searches by Kemp et al., reference [7], show that an auditory stimulus takes only 8-10 milliseconds to reach the brain, but on the other hand, a visual stimulus takes 20-40 milliseconds. This implies that the faster the stimulus reaches the motor cortex, faster will be the reaction time to the stimulus. Therefore since the auditory stimulus reaches the cortex faster than the visual stimulus, the auditory reaction time is faster than the visual reaction time.

Reaction times are widely used to evaluate neuromuscular-physiological responses in sports. Studies by Pain \& Hibbs, reference [1], have shown that the neuromuscular-physiological component of an auditory reaction time for sprint athletes can be around 85 milliseconds. Faster reaction times are significant for better performance of athletes. The faster the stimulus reaches the brain, the faster the signal is processed and the necessary responses are sent for the necessary motor reaction. Van den Berg et al., reference [8], also found that fatigue due to sleep dep- rivation caused subjects to have slower reaction times. Studies by Ando et al., reference [9], reported that reaction times reduced with repeated practice. Therefore reaction times to a particular stimulus can be made faster with repeated practice with a particular stimulus and with adequate rest in between stimuli.

In this study, as seen in Figure 2, it was also found that the male subjects had faster reaction times compared to the female subjects for both auditory as well as visual stimuli. This finding is similar to the research done by Dane et al., reference [6]. The reason for this difference

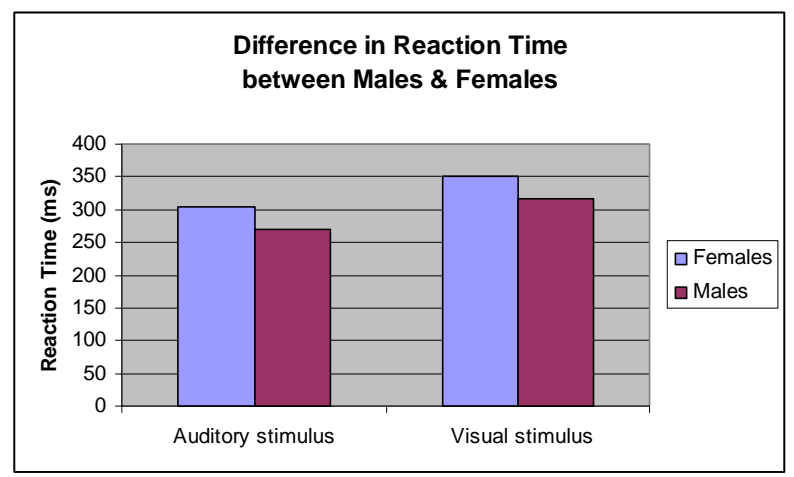


Figure 2. Graph showing males having faster simple reaction time compared to females for both auditory and visual stimuli.

could be that it takes the same time for both the auditory and visual stimuli to reach the cortex but the time taken for the corresponding motor response and muscle contrac- tion might differ. This was documented in the study done by Silverman, reference [10], that the motor response is faster in males when compared to females because they are comparatively stronger than females. This explains why males have faster simple reaction times for both auditory as well as visual stimuli.

\section{Conclusions}

From the above study it can be concluded that simple reaction time is faster for auditory stimuli compared to visual stimuli. Auditory stimuli has

- The fastest conduction time to the motor cortex.

- Fast processing time in the auditory cortex.

- Therefore faster reaction time and quick muscle contraction.

- And on the whole improves the performance of the athlete.

As exercise physiologists, our main aim is to improve the speed, skill and performance of the athlete. The above evidences suggest that speed and performance of an activity can be improved with faster reaction time to a stimulus. From the above findings of the study, faster reaction times can be achieved by providing repeated auditory stimuli and with adequate periods of rest between the stimuli.

A performance enhancing program can look like this:

- Exposure to adequate auditory stimuli;

- Repeated exposure to stimuli during practice;

- Adequate periods of rest between practices.

\section{REFERENCES}

[1] T. G. Matthew Pain and A. Hibbs, "Sprint Starts and the
Minimum Auditory Reaction Time," Journal of Sports Sciences, Vol. 25, No. 1, 2007, pp. 79-86.

[2] P. D. Thompson, J. G. Colebatch, P. Brown, J. C. Rothwell, B. L. Day and J. A. Obeso, "Voluntary Stimulus Sensitive Jerks and Jumps Mimicking Myoclonus or Pathological Startle Syndromes," Movement Disorders, Vol. 7, No. 3, 1992, pp. 257-262.

[3] Y. Yagi, K. L. Coburn, K. M. Estes and J. E. Arruda, "Effects of Aerobic Exercise and Gender on Visual and Auditory P300, Reaction Time, and Accuracy,” European Journal of Applied Physiology, Vol. 80, 1999, pp. 402408.

[4] R. Verlager, "On the Utility of P3 Latency as an Index of Mental Chronometry,” Journal of Psychophysiology, Vol. 34, No. 2, 1997, pp. 131-156.

[5] B. T. Engel, P. R. Thorne and R. E. Quilter, "On the Relationship Among Sex, Age, Response Mode, Cardiac Cycle Phase, Breathing Cycle Phase and Simple Reaction Time,” Journal of Gerontology, Vol. 27, No. 4, 1972, pp. 456-460.

[6] S. Dane and A. Erzurumluoglu, "Sex and Handedness Differences in Eye-Hand Visual Reaction Times in Handball Players," International Journal of Neuroscience, Vol. 113, No. 7, 2003, pp. 923-929.

[7] B. J. Kemp, "Reaction Time of Young and Elderly Subjects in Relation to Perceptual Deprivation and Signal-on Versus Signal-off Condition,” Developmental Psychology, Vol. 8, No. 2, 1973, pp. 268-272.

[8] B. J. Van den and G. Neely, "Performance on a Simple Reaction Time Task While Sleep Deprived,” Perceptual and Motor Skills, Vol. 102, No. 2, 2006, pp. 589-600.

[9] S. Ando, N. Kida and S. Oda, "Practice Effects on Reaction Time for Peripheral and Central Visual Fields," Perceptual and Motor Skills, Vol. 95, No. 3, 2002, pp. 747752.

[10] I. W. Silverman, "Sex Differences in Simple Visual Reaction Time: A Historical Meta-Analysis (Sports Events)," Sex Roles: A Journal of Research, Vol. 54, No. 1-2, 2006, pp. 57-69. 